\title{
Sleep Duration, Sleep Regularity, Body Weight, and Metabolic Homeostasis in School-aged Children
}

AUTHORS: Karen Spruyt, PhD, ${ }^{a}$ Dennis L. Molfese, PhD, ${ }^{b}$ and David Gozal, MDa

aDepartment of Pediatrics, Comer Children's Hospital and Pritzker School of Medicine, University of Chicago, Chicago, Illinois; and ${ }^{b}$ Birth Defects Center, University of Louisville, Louisville, Kentucky

KEY WORDS

sleep duration, obesity, children, lipids, insulin resistance, inflammation

\section{ABBREVIATIONS}

LDL-low-density lipoprotein

HDL—high-density lipoprotein

CRP-high sensitivity C-reactive protein

TST-total sleep time

$\mathrm{RDI}$-respiratory disturbance index

SV—sleep variability

www.pediatrics.org/cgi/doi/10.1542/peds.2010-0497

doi:10.1542/peds.2010-0497

Accepted for publication 0ct 19, 2010

Address correspondence to David Gozal, MD, University of Chicago, Comer Children's Hospital, Department of Pediatrics, 5721 S. Maryland Ave, MC 8000, Suite K-160, Chicago, IL 60637.

E-mail: dgozal@uchicago.edu

PEDIATRICS (ISSN Numbers: Print, 0031-4005; Online, 1098-4275).

Copyright (@) 2011 by the American Academy of Pediatrics

FINANCIAL DISCLOSURE: The authors have indicated they have no financial relationships relevant to this article to disclose.

Funded by the National Institutes of Health (NIH).

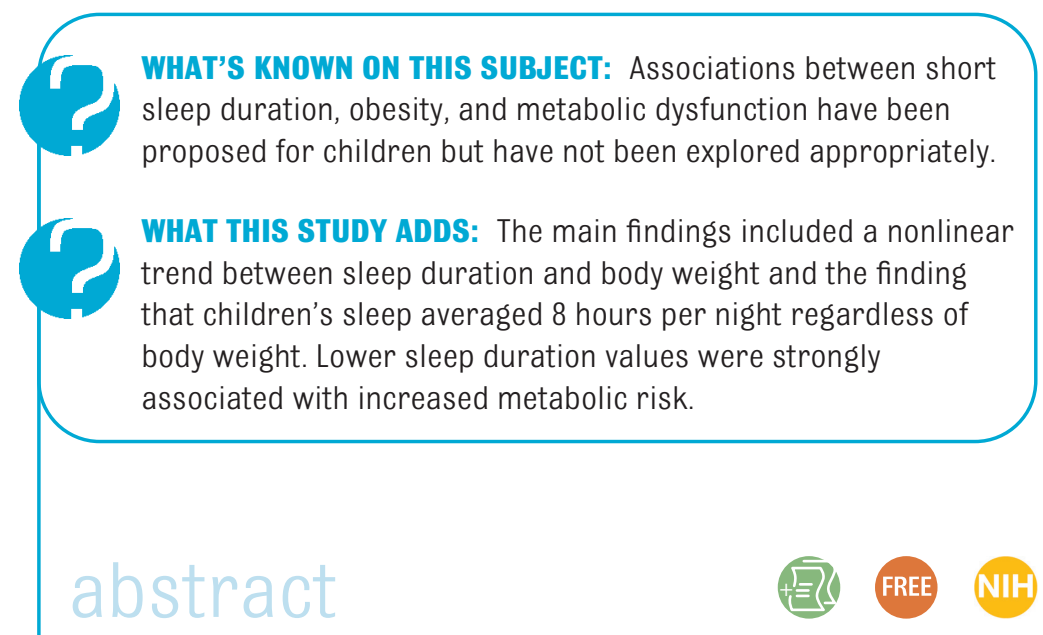

OBJECTIVE: The goal was to explore the effects of duration and regularity of sleep schedules on BMI and the impact on metabolic regulation in children.

METHODS: Sleep patterns of 308 community-recruited children 4 to 10 years of age were assessed with wrist actigraphs for 1 week in a cross-sectional study, along with BMI assessment. Fasting morning plasma levels of glucose, insulin, lipids, and high-sensitivity C-reactive protein also were measured for a subsample.

RESULTS: Children slept 8 hours per night, on average, regardless of their weight categorization. A nonlinear trend between sleep and weight emerged. For obese children, sleep duration was shorter and showed more variability on weekends, compared with school days. For overweight children, a mixed sleep pattern emerged. The presence of high variance in sleep duration or short sleep duration was more likely associated with altered insulin, low-density lipoprotein, and highsensitivity C-reactive protein plasma levels. Children whose sleep patterns were at the lower end of sleep duration, particularly in the presence of irregular sleep schedules, exhibited the greatest health risk.

CONCLUSIONS: Obese children were less likely to experience "catchup" sleep on weekends, and the combination of shorter sleep duration and more-variable sleep patterns was associated with adverse metabolic outcomes. Educational campaigns, aimed at families, regarding longer and more-regular sleep may promote decreases in obesity rates and may improve metabolic dysfunction trends in school-aged children. Pediatrics 2011;127:e345-e352 
Sleep duration has increasingly gained perceived importance as it relates to health outcomes such as cognitive and emotional functions and body weight. Sleep duration is determined by multiple factors, some of which (such as seasonality) may be difficult to control, ${ }^{1}$ whereas others are embedded within a familial or societal context and can be modified. The latter include bedtime, sleep time (shortened or fragmented), rising time, adjustment of sleep phase to underlying circadian rhythms, and external mandatory schedules (eg, school days versus weekend days). A recent shift from consistent patterns of sleep throughout the week and on weekends to sleeping patterns in which children extend sleep duration on weekends or holidays has been noted and possibly reflects concurrent changes in societal lifestyles. ${ }^{2,3}$

Societies have evolved with a more intense and demanding pace of life, ${ }^{4}$ which leads to substantial reductions in sleep duration, even for children. ${ }^{5,6}$ Concomitant with reductions in sleep duration, an increasing surge in the prevalence of obesity that starts in early childhood has emerged, ${ }^{7,8}$ which suggests the existence of an association between sleep duration and obesity. ${ }^{8}$ Overweight and obese children seem to have shorter sleep or moredisrupted sleep, and children who slept 8 to 10 hours per night were 3.45 to 4.9 times more likely to be overweight than children who sleep more. ${ }^{9-11}$ In addition, the effects of lifestyle-modified sleep, such as weekend and/or holiday sleep compensation leading to improvements in the risk of childhood weight problems, were reported recently. ${ }^{12}$ Inadequate sleep may mediate changes in the levels of some of the neuropeptides that regulate appetite, such as increased levels of ghrelin and reduced levels of leptin, leading to increased food intake and obesity. ${ }^{13}$ For children, the potential association between sleep duration and obesity has not been explored extensively; more importantly, assessments have relied primarily on subjective estimates of sleep duration.,14-16 Furthermore, the effects of sleep schedule regularity on $\mathrm{BMI}$ and the impact of specific sleep patterns on metabolic regulation in children are not known.

We hypothesized that "optimal sleep" would be protective against the occurrence of overweight/obesity in children, with favorable glycemic and lipid levels and lower plasma levels of inflammatory markers, such as highsensitivity C-reactive protein (CRP). We recruited a large, community-based, pediatric cohort, in which we assessed sleep duration and sleep patterns objectively and also measured metabolic indicators.

\section{METHODS}

\section{Subjects}

Children were recruited from the community through the Jefferson County Public School system and represented, in terms of socioeconomic status and race/ethnicity, the general population of the city of Louisville, Kentucky. Children were excluded if they had any chronic medical conditions, genetic craniofacial syndromes, or neurobehavioral disorders. This current study was approved by the University of Louisville human research committee, and informed consent was obtained from the legal caregiver of each participant.

\section{Measurements}

\section{Anthropometric Measurements}

Children were weighed (to $0.1 \mathrm{~kg}$ ) with a calibrated scale, and height was measured (to $0.1 \mathrm{~cm}$ ) with a stadiometer (Holtain, Crymych, United Kingdom). BMI values were calculated, and $\mathrm{BMI} z$ scores were computed by using Centers for Disease Control and Pre- vention 2000 growth standards and software (Epi Info [Centers for Disease Control and Prevention, Atlanta, GA]). BMl $z$ scores of $\geq 1.65$ were considered to fulfill the criterion for obesity, whereas BMI $z$ scores of $>1.04$ were defined as overweight.

\section{Actigraphic Measurements}

The Actiwatch 3.4 (MiniMitter Actiwatch64, Resperonics, Bend, OR) measures $28 \times 27 \times 10 \mathrm{~mm}$ and weighs $17.5 \mathrm{~g}$. For this actigraph brand, epoch registration of activity counts is determined through comparison, that is, counts for the epoch in question and those immediately surrounding that epoch are weighted with a threshold sensitivity value (activity count), which was originally set at 40 (default value, being medium sensitivity), as follows: score $=E_{-2}(1 / 25)+E_{-1}(1 / 5)+$ $\mathrm{E}_{0}+\mathrm{E}_{+1}(1 / 5)+\mathrm{E}_{+2}(1 / 25)$, with $E_{n}$ being activity counts for the epoch and $E_{0}$ the scored epoch.

Epochs with activity counts that were equal to or below the threshold sensitivity value were scored as sleep, whereas epochs with counts that exceeded the threshold sensitivity value were scored as awake. The actigraphic sleep interval was marked manually for each record on the basis of the sleep log bedtime and rising time. The actigraphic parameter of interest was total sleep time (TST), representing the amount of time between sleep start and sleep end scored as sleep. Sleep start and sleep end were determined automatically as the first and last 10minute periods, respectively, in which no more than 1 epoch was scored as mobile. The actigraphy algorithm enabled summation of the number of epochs that did not exceed the threshold sensitivity value; therefore, it provided individual TST values for each night of recording. Each child wore an actigraphic device on his or her nondominant wrist for a period of 1 week. Acti- 
graphic recordings are considered a valid means of sleep assessment with respect to sleep-wake patterns. ${ }^{17,18}$

\section{Blood Tests}

For a random subsample of children, the following metabolic markers were measured after an overnight fast: glucose, insulin, triglycerides, total cholesterol, high-density lipoprotein (HDL) cholesterol, low-density lipoprotein (LDL) cholesterol, and high-sensitivity CRP. Serum insulin levels were measured by using a commercially available radioimmunoassay kit (Coat-ACount Insulin [Diagnostic Products Corp, Los Angeles, CA]). Plasma glucose levels were measured by using a commercial kit based on the hexokinase-glucose-6-phosphate dehydrogenase method (Flex reagent cartridges [Dade Behring, Newark, DE]). Serum lipid levels, including total cholesterol, HDL cholesterol, calculated LDL cholesterol, and triglyceride levels, were assessed by using Flex reagent cartridges (Dade Behring). Serum high-sensitivity CRP concentrations were measured within 2 to 3 hours by using Flex reagent cartridges (Dade Behring).

\section{Statistical Analyses}

Mean TST for 7 consecutive days, mean TST for Sunday through Thursday (ie, school days), and mean TST for Friday and Saturday (ie, weekend days) were calculated. Results are reported as means \pm SDs. Box-plots were generated for illustration of sleep patterns.
The potential dispersion of data points around the mean was expressed as the coefficient of variation or as individual sleep variability (SV) (ie, percentage SV relative to the mean TST). Sleep duration trends were calculated as the "moving average," with the average TST for 1 week being the baseline TST from which daily average changes were derived. Correlational analyses assessed the strength of potential relationships between sleep-related variables and $\mathrm{BMI} z$ scores and plasma assay results. All analyses were performed with Statistica 9.0 (Statsoft, Tulsa, OK).

\section{RESULTS}

In the first round of analyses, we analyzed the weight-sleep-biomarker interrelationships from a weight perspective. The median respiratory disturbance index (RDI) was 0.7 (interquartile range: $0.38-1.4)$, and values were significantly higher for obese children (normal weight, $0.9 \pm 1.9$; overweight, $0.8 \pm 1.3$; obese, $2.4 \pm 4.5$; $\left.\mathrm{F}_{2,244}=6.2 ; P=.003\right)$. The groups were comparable in their distribution according to gender and race/ethnicity, but the obese group was slightly older; therefore, when applicable, the subsequent analyses included age as a covariate (Table 1).

TST fluctuated around 8 hours, irrespective of school day or weekend day. However, substantial variability emerged in TST (Supplemental Table 5 and Fig 1). Among normal-weight, over- weight, and obese groups, comparisons of daily TST values showed no significant differences either for weekdays or for weekends (Supplemental Table 5). However, analysis of SV values within BMI $z$ score-defined groups revealed that, for obese children, TST on weekends was more variable than that on school days $\left(t_{195}=2.4 ; P=\right.$ $.016)$, compared with children with normal BMI $z$ scores $\left(t_{296}=-1.30\right.$; $P=.20)$ or overweight children $\left(t_{102}=\right.$ $0.68 ; P=.49$ ), for whom sleep duration on school days versus weekends was rather stable (Supplemental Table 5). Daily TST comparisons across the 3 BMI $z$ score-defined groups revealed no significant differences, but TST on weekend days was shorter for the obese children (analysis of covariance, $P=.03$ ) (Fig 1 and Supplemental Table 5).

A moving average plot depicting the trend characteristics of sleep duration (or SV) for each of the 3 weight groups is shown in Fig 2. Normal-weight children displayed rather regular sleep duration during the week, with a tendency for longer sleep on weekends. In contrast, although sleep duration seemed regular on school days for obese children, sleep duration became increasingly shorter as the week progressed, reaching maximal differences on the weekend. For overweight children, a mixed pattern emerged; these children slept longer as the week progressed and further prolonged their sleep over the weekend. A

TABLE 1 Age, Gender, and Race/Ethnicity Distributions Among Normal-Weight, Overweight, and Obese Children

\begin{tabular}{|c|c|c|c|c|c|c|}
\hline & Total & Normal-Weight & Overweight & Obese & $\begin{array}{c}\text { Test } \\
\text { Statistic }\end{array}$ & $P$ \\
\hline Age, mean $\pm S D, y$ & $7.2 \pm 1.3$ & $6.88 \pm 1.2$ & $6.99 \pm 1.3$ & $7.74 \pm 1.3$ & $F_{2,307}=14.9$ & .000001 \\
\hline Female, $\%$ & 51 & 24.4 & 9.42 & 17.21 & $\chi_{2}^{2}=0.9$ & .645 \\
\hline Race/ethnicity, \% (n) & & & & & $\chi_{2}^{4}=4$ & .406 \\
\hline Non-Hispanic white & $71.4(220)$ & 36.7 (113) & $13.3(41)$ & $21.4(66)$ & & \\
\hline Black & $18.8(58)$ & $9.1(28)$ & $2(6)$ & $7.8(24)$ & & \\
\hline Other & $9.7(30)$ & $4.6(14)$ & $2(6)$ & $3.3(10)$ & & \\
\hline BMI z score & & $0.07 \pm 0.7(N=155)$ & $1.37 \pm 0.17(N=53)$ & $2.33 \pm 0.38(N=100)$ & & \\
\hline
\end{tabular}

The age range was 4.1 to 10.1 years (95\% confidence interval: $7.0-7.3$ years); $15.6 \%$ of subjects were $<6$ years of age. 


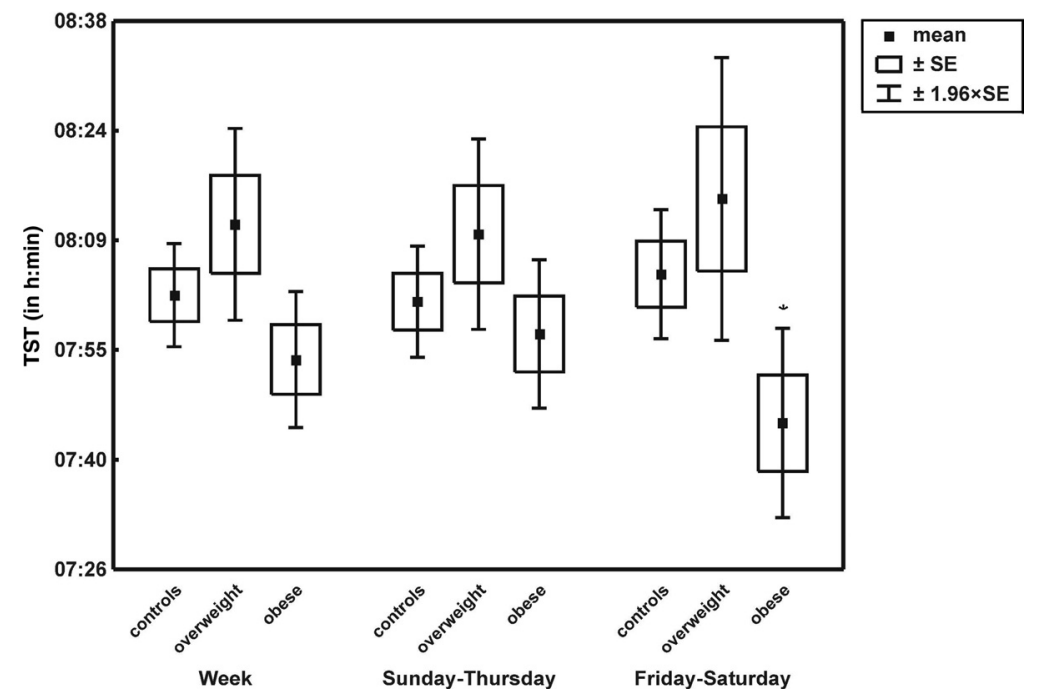

FIGURE 1

Mean TSTs for normal-weight, overweight, and obese children. ${ }^{*} P<.05$.
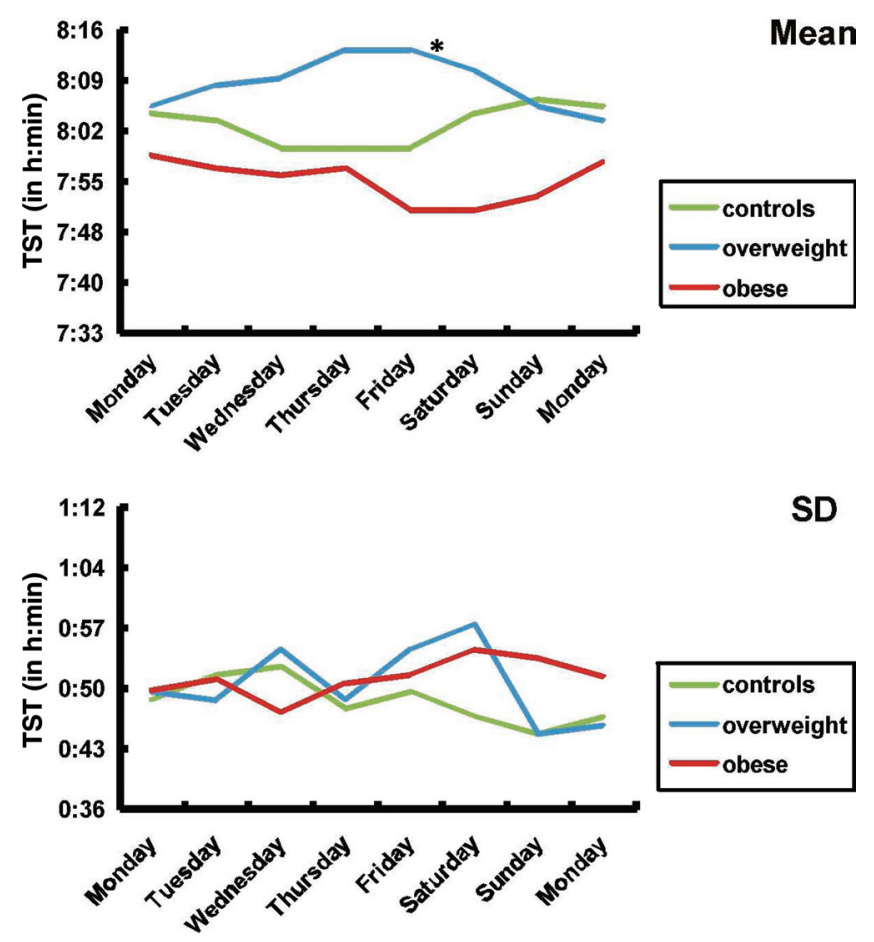

FIGURE 2

One-week trends of sleep durations for normal-weight, overweight, and obese children. ${ }^{*} P<.05$

group-day effect $\left(\mathrm{F}_{14,2135}=2.07 ; P=\right.$ .01) was found, in which Friday night was the turning point $\left(\mathrm{F}_{2,304}=3.7 ; P=\right.$ .03), with the overweight and obese children being radically different from each other (Fig 2). Table 2 shows the mean levels of the metabolic markers for the normal-weight, overweight, and obese groups.

BMI $z$ scores were not partially correlated (age as covariate) with sleep duration for normal-weight or obese children, but a significant association occurred for overweight chil- dren (school days: $r=0.31 ; P<.05$; weekend days: $r=0.29 ; P<.05$ ) (Supplemental Table 6).

Metabolic marker levels were partially correlated (age as covariate) with sleep duration for the normal-weight, overweight, and obese groups. For obese children, the variability in sleep duration on school days was positively correlated with triglyceride levels ( $r=$ $0.31 ; P<.05)$, with no other significant associations being found (Supplemental Table 7).

In a second round of analyses, we analyzed the weight-sleep-biomarker level interrelationships from a sleep perspective. All children were regrouped on the basis of their sleep patterns after we standardized TST with adjustment for age (ie, created TST $z$ scores for our sample), thus controlling for potential age fluctuations, to determine a cutoff value for TST at which health is at risk (eg, analogous to a BMI $z$ score). We used 3 cutoff values that is, 1 SD, $1.5 \mathrm{SDs}$, and $2 \mathrm{SDs}$ above and below the mean sleep duration for weekdays and weekends. Accordingly, 9 sleep pattern groups could be defined (Supplemental Fig 3 and Supplemental Table 9).

Gender distributions over the sleep pattern groups were equal, irrespective of the 3 cutoff approaches. Age differences were found between the NS and NN groups with the 1-SD cutoff value, with the latter being younger $\left(\mathrm{F}_{8,297}=2.1 ; P=.04\right)$, and between the NS and LN groups with the 1.5-SD cutoff value, with the latter being younger $\left(\mathrm{F}_{6,299}=2.9 ; P=.01\right.$ ) (Supplemental Table 8). Furthermore, with respect to race/ethnicity distribution, there was a trend toward significantly more black children in the SS and LS groups with the 1-SD $\left(\chi_{16}^{2}=32.7 ; P=.008\right)$ and 1.5-SD ( $\left.\chi_{12}^{2}=21.4 ; P=.04\right)$ cutoff values. RDI values were not different among sleep profile groups (Supplemental Table 8). No statistical differ- 
TABLE 2 Metabolic Marker Levels Among Normal-Weight, Overweight, and Obese Children

\begin{tabular}{|c|c|c|c|c|c|}
\hline & \multicolumn{3}{|c|}{ Level, Mean \pm SD } & \multirow[t]{2}{*}{$\mathrm{F}_{2,106}$} & \multirow[t]{2}{*}{$P$} \\
\hline & Normal-Weight & Overweight & Obese & & \\
\hline Glucose & $75.9 \pm 16.4$ & $78.9 \pm 16.8$ & $83.7 \pm 13.0$ & 2.7 & .07 \\
\hline Insulin & $4.5 \pm 3.4$ & $6.4 \pm 5.3$ & $11.5 \pm 7.3$ & 16.7 & $.000001^{\mathrm{a}}$ \\
\hline Triglycerides & $67.9 \pm 40.7$ & $80.3 \pm 33.1$ & $85.7 \pm 37.7$ & 7.0 & $.002^{\mathrm{a}}$ \\
\hline Total cholesterol & $157.2 \pm 25.2$ & $168.3 \pm 31.1$ & $161.6 \pm 28$ & 2.6 & .08 \\
\hline $\mathrm{HDL}$ & $53.4 \pm 10.7$ & $50.5 \pm 10.1$ & $47.4 \pm 9.7$ & 3.0 & .05 \\
\hline LDL & $90.3 \pm 21.9$ & $101.7 \pm 24.2$ & $97.0 \pm 23.5$ & 3.8 & $.02^{\mathrm{a}}$ \\
\hline CRP & $1.3 \pm 1.3$ & $1.1 \pm 1.4$ & $3.9 \pm 4.9$ & 7.4 & $.001^{\mathrm{a}}$ \\
\hline
\end{tabular}

The characteristics of the subsample $(N=132)$ of children who agreed to have a blood sample drawn were similar to those of the children who refused and were as follows: $45.5 \%$ female, $66.7 \%$ non-Hispanic white, $19.7 \%$ black, $13.6 \%$ other race; age (mean \pm SD): $7.7 \pm 1.2$ years ( $95 \%$ confidence interval: $7.5-7.9$ years); BMl z score: $1.2 \pm 1.3$ ( $95 \%$ confidence interval: 1-1.4); RDI: $2.1 \pm 4$ (95\% confidence interval: 1.4-2.8); weekly TST: $467 \pm 48$ minutes; TST on weekdays: $470 \pm 51$ minutes; TST on weekend days: $463 \pm 68$ minutes.

a Significant $P$ value.

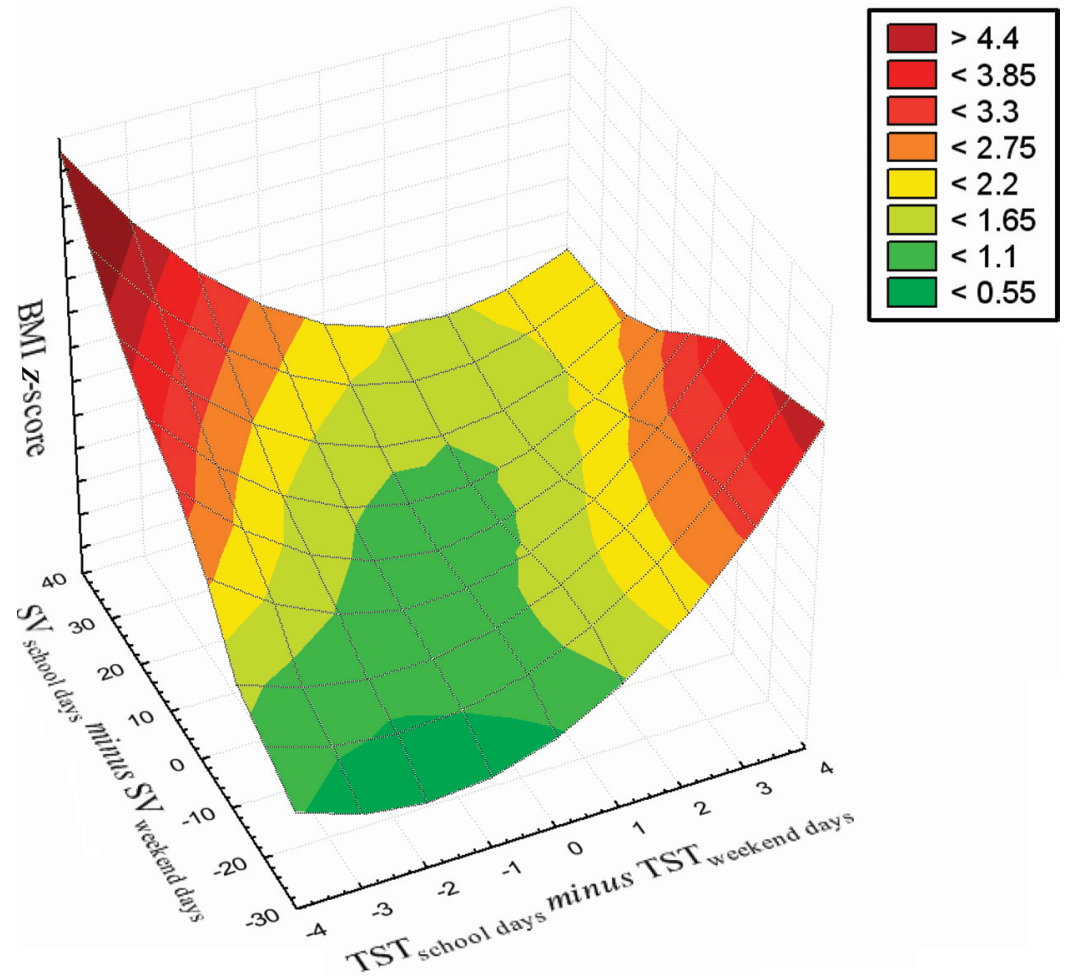

FIGURE 3

Surface plot depicting interrelationships between BMI $z$ scores, SV values, and TST values for 308 community children.

ences among sleep pattern groups were found in BMI $z$ scores (Supplemental Table 8), with similar variances in BMI z scores occurring for the sleep pattern groups. Finally, to illustrate further the interactions between BMI $z$ scores, sleep duration on school days and weekend days, and the effect of SV, a surface plot that depicts the complex interrelationships between BMI $z$ scores and sleep patterns was constructed (Fig 4).

Close inspection of the metabolic variables in the context of the 9 sleep subgroups, as defined above, suggested that optimal sleep was best represented by the LL group. With this latter group as the reference, LN, SN, and SS groups demonstrated significant associations with insulin, LDL, and CRP levels (Table 3).
On the basis of the observation that BMI $z$ scores and metabolic marker levels were not associated in the LL group, this group was defined as the reference group (Table 4). BMI $z$ scores were moderately correlated with metabolic marker levels in the NN group, irrespective of the SD cutoff values used. Similarly, BMI $z$ scores was significantly associated with CRP levels and insulin levels in the SN and SS groups (Table 4).

\section{DISCUSSION}

The study shows that community children 4 to 10 years of age routinely sleep $\sim 8$ hours per night, markedly below the current recommendations. ${ }^{19}$ Furthermore, the mean objective durations of sleep for normal-weight, overweight, and obese children did not differ significantly. However, the variation in sleep duration on school days and weekends was significantly different for obese children, and shorter sleep duration on weekends was more commonly found among obese children. Finally, although obesity is clearly a risk factor for metabolic dysfunction, ${ }^{20,21}$ a high variance in nightto-night sleep duration or short sleep duration was more likely to be associated with altered metabolic marker levels. In other words, the longer and more-stable sleep duration is, the less likely a child is to manifest metabolic dysfunction.

Some study limitations need to be addressed. Sleep duration measurements were performed by using actigraphy for 1 week, representing a typical school week, and BMI z scores represented single measurements. Therefore, longitudinal measurements over longer periods of time that include holidays and vacations might reveal a more-accurate predictive pattern that might improve some of the associations identified in this study. In addition, only BMI values and no other 
TABLE 3 Correlations Between Mean Weekly Sleep Duration and Metabolic Marker Levels for 9 Sleep Pattern Subgroups

\begin{tabular}{|c|c|c|c|c|c|c|c|}
\hline \multirow[t]{2}{*}{ Subgroup } & \multicolumn{7}{|c|}{ Correlation With TSTs } \\
\hline & $\begin{array}{c}\text { Glucose } \\
\text { Level }\end{array}$ & $\begin{array}{c}\text { Insulin } \\
\text { Level }\end{array}$ & $\begin{array}{c}\text { Triglyceride } \\
\text { Level }\end{array}$ & $\begin{array}{c}\text { Cholesterol } \\
\text { Level }\end{array}$ & HDL Level & LDL Level & CRP Level \\
\hline \multicolumn{8}{|l|}{$1 \mathrm{SD}$} \\
\hline SS & 0.08 & -0.43 & -0.19 & -0.26 & 0.25 & -0.44 & $-0.63^{a}$ \\
\hline SN & 0.33 & 0.12 & -0.18 & $-0.60^{\mathrm{a}}$ & -0.06 & $-0.61^{\mathrm{a}}$ & 0.25 \\
\hline SL & - & - & 0.96 & 0.97 & 0.41 & 0.87 & - \\
\hline NS & -0.33 & -0.44 & -0.36 & -0.03 & -0.03 & 0.08 & -0.43 \\
\hline NN & $0.38^{\mathrm{b}}$ & -0.05 & -0.003 & -0.06 & -0.02 & -0.06 & -0.02 \\
\hline NL & 0.09 & 0.31 & -0.48 & 0.05 & 0.22 & 0.09 & 0.17 \\
\hline LS & - & - & - & - & - & - & - \\
\hline LN & 0.64 & $0.71^{\mathrm{a}}$ & 0.23 & -0.27 & 0.04 & -0.40 & $0.82^{\mathrm{b}}$ \\
\hline $\mathrm{LL}$ & 0.19 & - & 0.60 & 0.64 & -0.11 & -0.23 & -0.05 \\
\hline \multicolumn{8}{|l|}{$1.5 \mathrm{SDs}$} \\
\hline SS & -0.14 & -0.38 & -0.03 & 0.07 & 0.29 & -0.09 & -0.48 \\
\hline SN & 0.12 & -0.03 & -0.24 & -0.36 & 0.26 & -0.44 & 0.50 \\
\hline NS & -0.03 & -0.12 & $-0.73^{a}$ & -0.13 & -0.34 & 0.30 & -0.06 \\
\hline NN & 0.11 & 0.04 & -0.05 & 0.09 & -0.04 & 0.14 & 0.002 \\
\hline NL & 0.89 & 0.55 & 0.23 & $0.92^{\mathrm{a}}$ & 0.62 & 0.81 & 0.03 \\
\hline LN & 0.76 & $0.96^{\mathrm{a}}$ & 0.77 & -0.30 & -0.90 & -0.52 & $0.99^{\mathrm{a}}$ \\
\hline $\mathrm{LL}$ & 0.19 & - & 0.60 & 0.64 & -0.11 & -0.29 & -0.05 \\
\hline \multicolumn{8}{|l|}{2 SDs } \\
\hline SN & -0.41 & -0.16 & -0.16 & -0.28 & 0.17 & -0.31 & 0.61 \\
\hline NS & -0.04 & -0.45 & -0.18 & 0.61 & 0.77 & 0.59 & -0.67 \\
\hline NN & 0.10 & -0.04 & 0.005 & 0.15 & 0.07 & 0.15 & -0.01 \\
\hline $\mathrm{NL}$ & - & - & - & - & - & - & - \\
\hline
\end{tabular}

SS indicates short sleep duration on schooldays and short sleep duration on weekend days (ie, consistently short sleepers); SN, short sleep duration on schooldays and normal sleep duration on weekend days (with normal being the standard of the community sample); SL, short sleep duration on schooldays and long sleep duration on weekend days; NS, normal sleep duration on schooldays and short sleep duration on weekend days; NN, normal sleep duration on schooldays and normal sleep duration on weekend days (ie, consistently normal sleepers); NL, normal sleep duration on schooldays and long sleep duration on weekend days; LS, Iong sleep duration on schooldays and short sleep duration on weekend days; LN, Iong sleep duration on schooldays and normal sleep duration on weekend days; LL, Iong sleep duration on schooldays and long sleep duration on weekend days (ie, consistently long sleepers). For illustration purposes, sleep duration for the SS group was $\sim 6.5$ hours, that for the NN group was $\sim 8$ hours, and that for the LL group was $\sim 9.5$ hours (Supplemental Tables 8 and 9). - indicates insufficient cases.

a Significant at $P<.05$

b Significant at $P<.01$

body fat distribution measurements (ie, visceral and subcutaneous fat amounts) were obtained. Furthermore, the sample was predominantly white non-Hispanic, and inferences with respect to other $\mathrm{racial} / \mathrm{ethnic}$ groups are not possible. Lastly, although there were no apparent differences between children for whom metabolic marker measurements were obtained versus not obtained, only $36.6 \%$ of the children had blood samples drawn.

Considering the reported trends indicating that children sleep progressively less, ${ }^{22}$ an alarming aspect is that, when children are given the opportunity to sleep they tend to sleep more, as observed on weekends and holidays, and such compensatory sleep seems to mitigate some of the adverse effects of weekday sleep patterns. 10,12 These observations prompt questions regarding the interrelationships of lifestyle-modified sleep and the obesity epidemic. Many studies have relied on parental reports, which generally overestimate children's sleep. ${ }^{16-18}$ In reviews of Iongitudinal and cross-sectional studies that examined the association between sleep duration and obesity in children, $14,15,23,24$ the scarcity of objective sleep measurements in pediatric populations is apparent. Only 2 studies used actigraphic sleep measurements, and they limited their recordings to 24 hours. ${ }^{1,25}$ On the basis of our 24-hour/7-day recording approach, we show that, in addition to sleep duration, SV needs to be incorporated into analyses of sleepbody weight relationships. The complexity of these relationships is best illustrated in Fig 3, which corroborates the "variable consistency in existing data"14 and addresses whether SV is a risk factor for obesity. ${ }^{14}$ The scarcity of Iongitudinal studies and the disparity in age ranges across studies and within studies compound the existing limitations of current knowledge. $5,7,12,13,19,20$ Cappuccio et $\mathrm{al}^{24}$ addressed the potential confounders in existing studies (eg, gender proportions, sample size, and sampling) but applied a very different cutoff value for defining short sleep for children, compared with Chen et al, ${ }^{16}$ that is, $\leq 10$ hours per night, which is at odds with current findings in a representative pediatric US population. The heterogeneity of the relationship between sleep duration and obesity reported in the various published studies is remarkable, from an absence of any association to a negative linear trend or an n-shaped relationship. Such discrepancies might simply reflect sampling bias or overcontrolling for certain variables. Several studies indicated that sleep duration and body weight are determined by a multitude of factors, such as sociodemographic, socioeconomic, familial (eg, family structure and overweight parent), and individual (eg, health behavior and health status) characteristics. ${ }^{5,26-28}$ Therefore, we should incorporate biases, confounders, and covariates in the interpretation of small variances. ${ }^{29,30}$ In addition, for a full understanding of sleep duration-body weight relationships, the entire body weight spectrum 
TABLE 4 Correlations Between Metabolic Marker Levels and BMI z Scores for 9 Sleep Pattern Subgroups

\begin{tabular}{|c|c|c|c|c|c|c|c|}
\hline \multirow[t]{2}{*}{ Subgroup } & \multicolumn{7}{|c|}{ Correlation With BMI z Scores } \\
\hline & $\begin{array}{c}\text { Glucose } \\
\text { Level }\end{array}$ & $\begin{array}{l}\text { Insulin } \\
\text { Level }\end{array}$ & $\begin{array}{c}\text { Triglyceride } \\
\text { Level }\end{array}$ & $\begin{array}{c}\text { Cholesterol } \\
\text { Level }\end{array}$ & HDL Level & LDL Level & CRP Level \\
\hline \multicolumn{8}{|l|}{$1 \mathrm{SD}$} \\
\hline SS & 0.33 & 0.52 & 0.37 & 0.23 & -0.55 & 0.38 & $0.70^{\mathrm{a}}$ \\
\hline SN & 0.41 & $0.85^{\mathrm{a}}$ & 0.15 & 0.16 & -0.18 & 0.20 & 0.54 \\
\hline SL & - & - & 0.99 & 0.92 & 0.52 & 0.79 & - \\
\hline NS & 0.23 & 0.46 & 0.02 & -0.14 & -0.20 & -0.06 & 0.49 \\
\hline NN & 0.14 & $0.52^{\mathrm{b}}$ & $0.34^{\mathrm{c}}$ & 0.20 & -0.17 & 0.25 & $0.35^{\mathrm{c}}$ \\
\hline $\mathrm{NL}$ & -0.01 & 0.51 & 0.19 & -0.18 & $-0.78^{a}$ & 0.09 & 0.49 \\
\hline LS & - & - & - & - & - & - & - \\
\hline LN & 0.61 & 0.58 & 0.58 & -0.41 & -0.57 & -0.55 & 0.55 \\
\hline $\mathrm{LL}$ & -0.71 & - & -0.95 & -0.10 & 0.65 & 0.78 & -0.52 \\
\hline \multicolumn{8}{|l|}{$1.5 \mathrm{SDs}$} \\
\hline SS & 0.53 & 0.62 & 0.45 & 0.32 & $-0.77^{\mathrm{c}}$ & 0.60 & $0.76^{c}$ \\
\hline SN & 0.19 & $0.76^{\mathrm{a}}$ & 0.53 & 0.42 & -0.01 & 0.37 & $0.69^{c}$ \\
\hline NS & 0.47 & 0.37 & 0.05 & 0.47 & 0.14 & 0.54 & 0.41 \\
\hline NN & 0.10 & $0.53^{\mathrm{b}}$ & $0.24^{\mathrm{c}}$ & 0.08 & $-0.24^{c}$ & 0.14 & $0.35^{\mathrm{a}}$ \\
\hline $\mathrm{NL}$ & -0.45 & 0.09 & 0.53 & -0.61 & -0.80 & -0.67 & -0.41 \\
\hline LN & $0.99^{c}$ & 0.59 & 0.73 & -0.53 & -0.87 & -0.74 & 0.82 \\
\hline $\mathrm{LL}$ & -0.71 & - & -0.95 & -0.10 & 0.65 & 0.78 & -0.52 \\
\hline \multicolumn{8}{|l|}{2 SDs } \\
\hline SN & -0.03 & 0.71 & 0.79 & 0.55 & -0.08 & 0.46 & 0.24 \\
\hline NS & 0.38 & 0.49 & -0.10 & -0.36 & -0.40 & -0.19 & 0.67 \\
\hline NN & 0.18 & $0.50^{\mathrm{b}}$ & $0.24^{c}$ & 0.07 & $-0.25^{a}$ & 0.13 & $0.36^{\mathrm{b}}$ \\
\hline $\mathrm{NL}$ & - & - & - & - & - & - & - \\
\hline LN & - & - & - & - & - & - & - \\
\hline
\end{tabular}

Insulin levels were significantly different across 4 of the subgroups only (for $2 \mathrm{SDs}, \mathrm{F}_{5,104}=3.03 ; P=.01$; SN group, $5.5 \pm$ $4.6 \mu \mathrm{U} / \mathrm{mL}$; NS group, $15.4 \pm 11.1 \mu \mathrm{U} / \mathrm{mL}$; NN group, $7.7 \pm 5.5 \mu \mathrm{U} / \mathrm{mL}$; LN group, $16.5 \pm 20.5 \mu \mathrm{U} / \mathrm{mL}$ ) and triglyceride levels showed similar findings (for $2 \mathrm{SDs}, \mathrm{F}_{5,104}=3.7 ; P=.004$; SN group, $57.8 \pm 32.3 \mathrm{mg} / \mathrm{dL}$; NS group, $115.3 \pm 64.2 \mathrm{mg} / \mathrm{dL}$; NN group, $74.2 \pm 29.8 \mathrm{mg} / \mathrm{dL} ;$ LN group, $105 \pm 41.01 \mathrm{mg} / \mathrm{dL}$ ). — indicates insufficient cases.

a Significant at $P<.01$.

b Significant at $P<.001$.

c Significant at $P<.05$

should be included, even failure to thrive. We have analyzed the same sample from 2 perspectives, that is, on the basis of weight and sleep, and have expressed this interrelationship in a metric fashion, as well as associating it with metabolic homeostasis.

Regardless of previous limitations, the association of sleep duration with BMI among children putatively showed $\sim 1.5$ to 2 -fold increases (odds ratio: $1.15-11)$ in the odds of being a short sleeper if the child was obese, ${ }^{16,24,31,32}$ a trend that we confirmed (Fig 3). Similar to the results reported by Wing et $\mathrm{al}^{12}$ the compensatory effect of longer sleep on weekends may partially ameliorate the risk of childhood overweight/obesity and overall may benefit health. Among our subgroups, there was an $\sim 1.5$-hour difference in sleep duration between the $\mathrm{NN}$ and $\mathrm{LL}$ groups, and the BMI $z$ score distributions agreed closely with the findings reported by Chen et al, ${ }^{16}$ who suggested a 9\% decrease in the risk of increased body weight through sleep prolongation by 1 hour per night. 0 n the basis of the global differences between obese, overweight, and normalweight groups (Fig 2), a sleep extension of even $\sim 30$ minutes per night might have such desirable effects on $\mathrm{BMI}$ and metabolic regulation and would be more likely to be implemented consistently in a family setting, as shown by Sadeh et al..$^{33}$ Our results on sleep distribution patterns corrob- orate those of Touchette et al, ${ }^{34}$ who showed that sleep patterns and sleep durations seemed to be preserved consistently across long periods of time during childhood and that the effects on BMI $z$ scores and metabolic marker levels of the adversely associated sleep patterns identified in the present study might be sustained for many years and might become difficult to reverse if interventions are not implemented early in life.

Although information on sleep duration is lacking, a longitudinal trend in obesity has been identified, with approximately 1 in 3 obese preschoolaged children and approximately 1 in 2 obese school-aged children becoming obese adults. ${ }^{7,35}$ Therefore, identification of children at risk during infancy and early childhood and implementation of prospective interventions aiming to prolong and to regularize sleep for such children should provide more-definitive answers regarding the role of sleep in the context of BMI regulation and metabolic homeostasis. Indeed, sleep patterns at the lower end of sleep duration, particularly in the presence of irregularity, were strongly associated with increased health risk, as evidenced by increased BMI $z$ scores and adverse changes in fasting insulin, LDL cholesterol, and CRP plasma concentrations. Irregular, unstable, and inadequately short sleep durations may potentially trigger inflammatory pathways and exert adverse effects on insulin sensitivity and lipids in the presence of obesity.

\section{CONCLUSIONS}

Community children in the United States exhibited much shorter objective sleep durations than previously anticipated. Although obese children did not display radically different total weekly sleep durations, compared with their normal-weight counterparts, analyses of SV throughout the 
week revealed that obese children were less likely to experience "catchup" sleep on weekends and the combination of shorter sleep duration and more-variable sleep patterns was associated with adverse metabolic outcomes. Therefore, public health campaigns aiming to educate families

\section{REFERENCES}

1. Nixon GM, Thompson JM, Han DY, et al. Short sleep duration in middle childhood: risk factors and consequences. Sleep. 2008; 31(1):71-78

2. Mindell JA, Meltzer LJ, Carskadon MA, Chervin RD. Developmental aspects of sleep hygiene: findings from the 2004 National Sleep Foundation Sleep in America Poll. Sleep Med. 2009;10(7):771-779

3. Acebo C, Sadeh A, Seifer R, Tzischinsky 0, Hafer A, Carskadon MA. Sleep/wake patterns derived from activity monitoring and maternal report for healthy 1- to 5-year-old children. Sleep. 2005;28(12):1568-1577

4. Lobstein T, Baur L, Uauy R. Obesity in children and young people: a crisis in public health. Obesity Rev. 2004;5(suppl 1):4-104

5. Krueger PM, Friedman EM. Sleep duration in the United States: a cross-sectional population-based study. Am J Epidemiol. 2009;169(9):1052-1063

6. National Sleep Foundation. Sleep in America Poll: sleep in children survey. Available at: www.sleepfoundation.org/article/sleepamerica-polls/2004-children-and-sleep. Accessed December 19, 2010

7. Wang Y, Beydoun MA. The obesity epidemic in the United States: gender, age, socioeconomic, racial/ethnic, and geographic characteristics: a systematic review and meta-regression analysis. Epidemiol Rev. 2007;29(1):6-28

8. Ogden CL, Flegal KM, Carroll MD, Johnson $\mathrm{CL}$. Prevalence and trends in overweight among US children and adolescents, 1999-2000. JAMA. 2002;288(14):1728-1732

9. Chaput JP, Brunet M, Tremblay A. Relationship between short sleeping hours and childhood overweight/obesity: results from the 'Quebec en Forme' Project. Int $J$ Obes (Lond). 2006;30 (7):1080-1085

10. Hart CN, Jelalian E. Shortened sleep duration is associated with pediatric overweight. Behav Sleep Med. 2008;6(4): 251-267

11. Locard E, Mamelle N, Billette A, Miginiac M, Munoz F, Rey S. Risk factors of obesity in a five year old population: parental versus en- regarding the benefits of longer and more-regular sleep may lead to decreased obesity and metabolic dysfunction trends for our children.

\section{ACKNOWLEDGMENTS}

This study was supported by National Institutes of Health grants HL65270 and HL70911 and a Comer Children's Hospital research award.

We acknowledge E. Dayyat, J. Bennett, and A. Roman for their assistance with actigraphic data collection and all of the families for their gracious participation. vironmental factors. Int J Obes Relat Metab Disord. 1992;16(10):721-729

12. Wing YK, Li SX, Li AM, Zhang J, Kong AP. The effect of weekend and holiday sleep compensation on childhood overweight and obesity. Pediatrics. 2009;124(5). Available at: www.pediatrics.org/ coil/content/full/124/5/e994

13. Zheng H, Berthoud H-R. Neural systems controlling the drive to eat: mind versus metabolism. Physiology. 2008;23(2):75-83

14. Marshall NS, Glozier N, Grunstein RR. Is sleep duration related to obesity? A critical review of the epidemiological evidence. Sleep Med Rev. 2008;12(4):289-298

15. Marshall NS, Glozier N, Grunstein RR. Reply to Taheri and Thomas: is sleep duration associated with obesity: $U$ cannot be serious. Sleep Med Rev. 2008;12(4):303-305

16. Chen X, Beydoun MA, Wang Y. Is sleep duration associated with childhood obesity? A systematic review and meta-analysis. Obesity (Silver Spring). 2008;16(2):265-274

17. Acebo C, LeBourgeois MK. Actigraphy. Respir Care Clin North Am. 2006;12(1):23-30, viii

18. Tryon WW. Issues of validity in actigraphic sleep assessment. Sleep. 2004;27(1):158-165

19. National Sleep Foundation. How much sleep do we really need? Available at: www. sleepfoundation.org/article/how-sleepworks/how-much-sleep-do-we-really-need. Accessed December 19, 2010

20. Van Cauter E, Knutson KL. Sleep and the epidemic of obesity in children and adults. Eur J Endocrinol. 2008;159(suppl 1):S59-S66

21. Butte NF, Cai G, Cole SA, et al. Metabolic and behavioral predictors of weight gain in Hispanic children: the Viva la Familia Study. Am J Clin Nutr. 2007;85(6):1478-1485

22. Iglowstein I, Jenni OG, Molinari L, Largo RH. Sleep duration from infancy to adolescence: reference values and generational trends. Pediatrics. 2003;111(2):302-307

23. Patel SR, Hu FB. Short sleep duration and weight gain: a systematic review. Obesity. 2008;16(3):643-653

24. Cappuccio FP, Taggart FM, Kandala NB, et al. Meta-analysis of short sleep duration and obesity in children and adults. Sleep. 2008; 31(5):619-626

25. Gupta NK, Mueller WH, Chan W, Meininger JC. Is obesity associated with poor sleep quality in adolescents? Am J Hum Biol. 2002; 14(6):762-768

26. Flynn MA, McNeil DA, Maloff B, et al. Reducing obesity and related chronic disease risk in children and youth: a synthesis of evidence with 'best practice' recommendations. Obes Rev. 2006;7 (suppl 1):7-66

27. Reilly JJ, Armstrong J, Dorosty AR, et al. Early life risk factors for obesity in childhood: cohort study. BMJ. 2005; 330(7504):1357-1359

28. Sekine M, Yamagami T, Handa K, et al. A dose-response relationship between short sleeping hours and childhood obesity: results of the Toyama Birth Cohort Study. Child Care Health Dev. 2002;28(2):163-170

29. Horne J. Short sleep is a questionable risk factor for obesity and related disorders: statistical versus clinical significance. Biol Psychol. 2008;77(3):266-276

30. Horne J. Too weighty a link between short sleep and obesity? Sleep. 2008;31(5): 595-596

31. Padez C, Mourao I, Moreira P, Rosado V. Long sleep duration and childhood overweight/obesity and body fat. Am J Hum Biol. 2009;21(3):371-376

32. Taveras EM, Rifas-Shiman SL, Oken E, Gunderson EP, Gillman MW. Short sleep duration in infancy and risk of childhood overweight. Arch Pediatr Adolesc Med. 2008;162(4):305-311

33. Sadeh A, Gruber R, Raviv A. The effects of sleep restriction and extension on schoolage children: what a difference an hour makes. Child Dev. 2003;74(2):444-455

34. Touchette E, Petit D, Tremblay RE, et al. Associations between sleep duration patterns and overweight/obesity at age 6. Sleep. 2008;31(11):1507-1514

35. Centers for Disease Control and Prevention. Obesity prevalence among low-income, preschool-aged children: United States, 1998-2008. MMWR Morb Mortal Wkly Rep. 2009;58(28):769-773 